\title{
A NEW SHORT-WAVELENGTH CARBON DIOXIDE BAND IN THE SPECTRUM OF VENUS
}

\author{
T. OWEN and H. P. MASON \\ IIT Research Institute, Chicago, Ill., U.S.A.
}

\begin{abstract}
In attempting to resolve the $\mathrm{CO}_{2}$ band at $7159 \AA$ identified in the spectrum of Venus by Spinrad (1962), we discovered a new band at $7105 \AA$. Both bands are fully resolved on our spectra and tentative $J$ numbers have been assigned to the rotational lines. In both cases, the $P$ branch appears to be anomalously weak. The $7159 \AA$ band has been observed in the laboratory (unresolved) by Herzberg and Herzberg (1953) at path lengths on the order of $55 \mathrm{~km}$ atm. To our knowledge, the $7105 \AA$ band has not been recorded previously. Following the predictions of Herman (1948) the two bands are given the assignments $5 v_{3}+2 v_{2}+v_{1}(7159 \AA)$ and $5 v_{3}+2 v_{1}(7105 \AA)$. An unsuccessful attempt was made to find the third member of this triad, $5 v_{3}+4 v_{2}$. In view of the very large amount of carbon dioxide apparently required to produce these absorptions, further study in the laboratory and at the telescope is encouraged.
\end{abstract}

\section{References}

Herman, R. C.: 1948, Astrophys. J. 107, 386.

Herzberg, G. and Herzberg, L.: 1953, J. Opt. Soc. Am. 43, 1037.

Spinrad, H.: 1962, Astrophys. J. 135, 651. 